\title{
Performance Analysis of Orthogonal Space Time Block Coding over Hoyt Fading Channels
}

\author{
George A. Ropokis, Athanasios A. Rontogiannis, and P. Takis Mathiopoulos \\ Institute for Space Applications and Remote Sensing, National Observatory of Athens, 15236, Athens, Greece \\ Email: \{ropokis,tronto,mathio\}@ space.noa.gr
}

\begin{abstract}
In this paper, a novel error probability analysis of orthogonal space-time block coding (OSTBC) over independent but not necessarily identical Hoyt (Nakagami- $q$ ) fading channels is presented. First, it is shown that for a precise performance analysis the derivation of the distribution of a quadratic form in normal random variables is necessary. After representing this distribution using a chi-squared series expansion a new, tight upper bound on the series truncation error is obtained. The proposed bound has a simple form, which allows the derivation of closed-form truncation error bound expressions for several performance analysis criteria. Based on the chi-squared series formulation, closed-form error probability expressions are also provided. Simulation results show that the proposed bounds are much tighter compared to previously derived ones and verify our analytical results.
\end{abstract}

\section{INTRODUCTION}

In recent years orthogonal space-time block coding (OSTBC) is becoming increasingly popular as an efficient transmit diversity technique to combat fading in wireless communications [1]. This approach offers full spatial diversity and maximum likelihood performance with linear decoding complexity. In the past, several performance analysis results have been reported for OSTBC operating over various classical fading channels, including Rayleigh, Nakagami-m [2],[3], and Nakagami-n (Rice) [4]. Another distribution, which has recently received increased attention in modelling fading channels is the Hoyt distribution. Several studies have shown that the Hoyt fading model provides a very accurate fit to experimental channel measurements in various telecommunications applications. For instance, in [5] this model has been used in outage analysis of cellular mobile radio systems, while in [6] a capacity analysis of Hoyt fading is provided. Similarly, the Hoyt distribution can be considered as an accurate fading model for satellite links with strong ionospheric scintillation [7]. However, regarding the performance of OSTBC technology over Hoyt fading channels, very few studies have been published in the open technical literature. These include [8], where an ergodic capacity analysis is presented, and [9] where the information outage probability of a OSTBC over Hoyt fading channels has been studied.

Motivated by the above, in this paper, the performance of OSTBC operating over independent but not necessarily

This paper is part of the 03ED838 research project, implemented within the framework of the "Reinforcement Programme of Human Research Manpower" (PENED) and co-financed by National and Community Funds (75\% from E.U.-European Social Fund and $25 \%$ from the Greek Ministry of Development-General Secretariat of Research and Technology). identical Hoyt distributed fading channels is studied. First, it is shown that for a precise analysis, the distribution of a positive definite quadratic form in normal random variables (RVs) must be determined. Among the several approaches (e.g. [10],[11],[12]) that have been proposed for the probability density function (PDF) of such a quadratic form we adopt the chi-squared expansion method, due to its simplicity, mathematical tractability and fast convergence. Then, a novel upper bound for the series expansion truncation error is derived. It turns out that this bound is much tighter than other previously derived bounds [10],[11]. The bound is also presented in a convenient simple form, which allows the derivation in closedform of various performance metrics truncation error bounds for OSTBC over Hoyt fading channels. Based on the chisquared series representation, novel closed-form expressions are also derived for the bit error probability (BEP) and symbol error probability (SEP) of various modulation schemes. It should be emphasized that the derivation of the PDF bound is quite general and can be applied to any fading model described by a positive definite quadratic form in normal RVs, as well as for other relevant applications [12].

The outline of this paper is as follows. In Section II, OSTBC over Hoyt fading is examined and its connection with quadratic forms is established. In Section III, the chi-squared expansion is defined and a new truncation error bound is derived. In Section IV, closed-form SEP and BEP expressions are given and error bounds on these expressions are provided. Simulation results are presented in Section V.

\section{Problem Formulation}

Let us consider an OSTBC multiple-input multiple-output (MIMO) system with $n_{t}$ transmit and $n_{r}$ receive antennae. It can be shown that the signal to noise ratio (SNR) per bit, $\gamma$, at the receiving end is expressed as [1]

$$
\gamma=\frac{\|\mathbf{H}\|_{F}^{2}}{R n_{t}} \gamma_{t}
$$

where $R$ is the rate of the space-time code and $\gamma_{t}$ is the SNR per bit at the transmitter side, defined as the ratio of the transmitted energy per bit over the noise power. In addition,

$$
\|\mathbf{H}\|_{F}^{2}=\sum_{p=1}^{n_{r}} \sum_{j=1}^{n_{t}}\left|h_{p j}\right|^{2}
$$

is the square of the Frobenius norm of the channel matrix, where $\left|h_{p j}\right|$ stands for the amplitude of the fading coefficient 
between the $p$ th receive and $j$ th transmit antenna. Assuming that $\left|h_{p j}\right|$ are independent Hoyt distributed RVs ${ }^{1}$, the PDF of the $\mathrm{RV} z_{p j}=\left|h_{p j}\right|^{2}$ is given by [13]

$p_{z_{p j}}(x)=\frac{\left(1+q_{p j}^{2}\right)}{2 q_{p j} \Omega_{p j}} \exp \left[-\frac{\left(1+q_{p j}^{2}\right)^{2} x}{4 q_{p j}^{2} \Omega_{p, j}}\right] I_{0}\left(\frac{\left(1-q_{p j}^{4}\right) x}{4 q_{p j}^{2} \Omega_{p j}}\right)$

for $x \geq 0$, where $\Omega_{p j}=E\left[\left|h_{p j}\right|^{2}\right], E[\cdot]$ being the expectation operator, $I_{0}(\cdot)$ is the zeroth order modified Bessel function of the first kind and $q_{p j}$ is the fading parameter of the corresponding Hoyt channel. It is known that a squared Hoyt RV can be expressed as the sum of squares of two independent zeromean normal RVs with different variances [14]. Therefore, for independent $\left|h_{p j}\right|$, (2) can be expressed as

$$
\|\mathbf{H}\|_{F}^{2} \stackrel{d}{=} \sum_{i=1}^{n} \sigma_{i}^{2} y_{i}^{2}
$$

where $n=2 n_{t} n_{r}, y_{i}$ 's are standard independent normal RVs, $\sigma_{i}$ 's are related to the parameters of the corresponding Hoyt $\mathrm{RVs}$ and $\stackrel{d}{=}$, denotes equality in distribution. Clearly, using (4), (1) can be written as

$$
\gamma \stackrel{d}{=} \sum_{i=1}^{n} a_{i} y_{i}^{2}
$$

with $a_{i}=\sigma_{i}^{2} \gamma_{t} / R n_{t}$. Hence, in order to obtain the performance criteria of OSTBC over Hoyt fading channels the PDF of $\gamma$, which is a positive definite quadratic form, must be properly defined. In the following section the required PDF is expressed based on a chi-squared series expansion and a very tight upper bound on the series truncation error is derived.

\section{TRUnCATION ERRor Bound FOR THE PDF OF A QUADRATIC FORM}

It is convenient to represent the right hand side (RHS) of (5) by the following RV

$$
Q=\sum_{i=1}^{n} a_{i} y_{i}^{2}
$$

Note that in our case $n$ is even, while $y_{i}, i=1,2, \ldots, n$, are zero mean, unit variance i.i.d. normal RVs, and $a_{i}$ are positive constants. In general, there is no known analytical expression for the PDF of $Q$. Instead, this PDF can be represented by an appropriate series expansion [12]. Using a chi-squared expansion, the PDF of $Q$ can be expressed as an infinite series [12, pp. 115 - 123]

$$
p_{Q}(x)=\sum_{k=0}^{\infty} c_{k} f(x, 2 \beta, n / 2+k)
$$

where

$$
f(x, b, l)=\frac{x^{l-1} \exp (-x / b)}{b^{l} \Gamma(l)}
$$

\footnotetext{
${ }^{1}$ It should be mentioned that the proposed analysis can be slightly modified to deal also with correlated Hoyt fading. However, due to space limitations this issue will not be further discussed here.
}

where $\Gamma(\cdot)$ is the gamma function and $\beta$ a parameter controling the convergence of (7). The coefficients $c_{k}$ of the expansion are given by

$$
c_{k}=c_{0} E\left[Z^{k}\right] /\left(2^{k} k !\right), \quad \text { with } \quad c_{0}=\prod_{i=1}^{n}\left(\beta / a_{i}\right)^{1 / 2}
$$

where $E\left[Z^{k}\right]$ is the $k$-th moment of a quadratic form $Z$ defined as

$$
Z \triangleq \sum_{i=1}^{n} \eta_{i} y_{i}^{2}, \quad \text { with } \quad \eta_{i}=1-\beta / a_{i} .
$$

Note that the convergence of the series (7) depends on the spread of the values $a_{i}$, i.e. on the ratio $\left[\max _{i}\left\{a_{i}\right\} / \min _{i}\left\{a_{i}\right\}\right]$ with small spreads leading to faster convergence. Assuming that the first $N+1$ terms are retained from the series in (7), in order to obtain a truncation error bound the following quantity must be properly bounded

$$
e(x)=\sum_{k=N+1}^{\infty}\left|c_{k}\right| f(x, 2 \beta, n / 2+k) .
$$

For this to happen, a bound on $\left|c_{k}\right|$ must be determined or equivalently from (9) the amplitude of the moments of $Z$ must be properly bounded. In [11], by defining the following quadratic form

$$
W=\sum_{i=1}^{n}\left|\eta_{i}\right| y_{i}^{2}
$$

and using the inequality

$$
\left|E\left[Z^{k}\right]\right| \leq E\left[W^{k}\right] \leq \max _{i}\left|\eta_{i}\right| E\left[\left(\sum_{i=1}^{n} y_{i}^{2}\right)^{k}\right]
$$

the following bound on $\left|c_{k}\right|$ has been obtained

$$
\left|c_{k}\right| \leq c_{0}\left(\max _{i}\left\{\left|\eta_{i}\right|\right\}\right)^{k} \Gamma(n / 2+k) /[k ! \Gamma(n / 2)] .
$$

However, performance evaluation results have shown that the bound obtained by the above inequality is rather loose (see Fig. 1). In order to derive a much tighter bound, it is noted that the number of terms $n$ is even, while $W$ can be written as follows

$$
W=\sum_{i=1}^{n} \lambda_{i} u_{i}^{2}
$$

where $\lambda_{i}$ and $u_{i}$ are proper permutations of $\left|\eta_{i}\right|$ and $y_{i}$ respectively, such that $\lambda_{i}$ are in decreasing order, for $i=1,2, \ldots, n$. The basic idea here is to formulate a new quadratic form which results from $W$ by grouping in pairs successive squared normal RVs $u_{i}^{2}$, i.e.,

$$
V=\sum_{i=1}^{n / 2} \lambda_{2 i-1}\left(u_{2 i-1}^{2}+u_{2 i}^{2}\right) .
$$

Comparing (15) and (16), clearly $W \leq V$ and thus $E\left[W^{k}\right] \leq$ $E\left[V^{k}\right] \forall k$. Furthemore and most importantly the moments 
of $V$ can be now easily obtained using its moment generating function (MGF)

$$
M_{V}(t)=\prod_{i=1}^{n / 2} \frac{1}{\left(1-2 \lambda_{2 i-1} t\right)} .
$$

By employing partial fractions and assuming that all $\lambda_{2 i-1}$ are distinct $^{2},(17)$, is expressed as:

$$
M_{V}(t)=\sum_{i=1}^{n / 2} A_{i} \frac{1}{\left(1-2 \lambda_{2 i-1} t\right)}
$$

where

$$
A_{i}=\lambda_{2 i-1}^{n / 2-1} \prod_{\substack{l=1 \\ l \neq i}}^{n / 2} \frac{1}{\lambda_{2 i-1}-\lambda_{2 l-1}} .
$$

Noting that (18) corresponds to the MGF of a RV that is a mixture of gamma distributed RVs, it can be shown that the moments of $V$ can be found as:

$$
E\left[V^{k}\right]=\sum_{i=1}^{n / 2} A_{i} k !\left(2 \lambda_{2 i-1}\right)^{k} .
$$

Since $\left|E\left[Z^{k}\right]\right| \leq E\left[W^{k}\right] \leq E\left[V^{k}\right]$, from (9),(19), and (20) the following very simple bound for $\left|c_{k}\right|$ can be obtained

$$
\left|c_{k}\right| \leq c_{0} \sum_{i=1}^{n / 2} \Delta_{i} \lambda_{2 i-1}^{n / 2+k-1}, \text { with } \Delta_{i}=\prod_{\substack{l=1 \\ l \neq i}}^{n / 2} \frac{1}{\lambda_{2 i-1}-\lambda_{2 l-1}}
$$

A tight truncation error bound, $e_{b}(x)$, can now be obtained by substituting (21) in the RHS of (11), i.e.,

$$
e_{b}(x)=\sum_{k=N+1}^{\infty} \sum_{i=1}^{n / 2} \Delta_{i} c_{0} \lambda_{2 i-1}^{n / 2+k-1} f(x, 2 \beta, n / 2+k) \text {. }
$$

Defining the series

$$
d(x, a, \beta) \triangleq \sum_{k=0}^{\infty} a^{k} f(x, 2 \beta, k+1)
$$

it is easily observed from (8) that (23) can be expressed as

$$
d(x, a, \beta)=\exp [-(1-a) x / 2 \beta] / 2 \beta .
$$

Furthermore, it can be proved that the series in (23) converges uniformly for $a>0$, as long as $a<1$. Hence, by selecting $\beta$ such that $\lambda_{2 i-1}<1$ for all $i$, the uniform convergence of (7) can be ensured and the truncation error bound is expressed in closed-form as

$$
e_{b}(x)=c_{0} \sum_{i=1}^{n / 2} \Delta_{i} g\left(x, \lambda_{2 i-1}, \beta, N+n / 2-1\right)
$$

where

$$
g(x, a, \beta, m)=d(x, a, \beta)-\sum_{k=0}^{m} a^{k} f(x, 2 \beta, k+1) .
$$

\footnotetext{
${ }^{2}$ This is a reasonable assumption since we have considered non-identically distributed Hoyt fading.
}

Notice that the expression of the truncation error comprises simple elementary functions only. It will become clear in the next section that this form of $e_{b}(x)$ dramatically simplifies the error probability performance analysis.

\section{ERROR PROBABILITY ANALYSIS}

In this section SEP and BEP closed-form expressions and error bounds are provided for PAM and QAM schemes employing OSTBC over Hoyt fading channels. For this purpose, integrals of the following form must be evaluated

$$
\mathcal{P}_{E}=\sum_{k=0}^{\infty} c_{k} \int_{0}^{\infty} f(\gamma, 2 \beta, n / 2+k) P(\gamma) d \gamma
$$

where $f(\cdot, \cdot, \cdot)$ is defined in (8), and $P(\cdot)$ is a function related to the modulation scheme. Additionally, using (25) bounds on the truncation error of the SEP and BEP series can be obtained by evaluating the integral

$$
\mathcal{E}=\int_{0}^{\infty} e_{b}(\gamma) P(\gamma) d \gamma
$$

\section{A. SEP Expressions}

Having obtained the PDF of $\gamma$ the SEP for several modulation schemes can be easily obtained, as follows:

1) $M$-PAM SEP: For an $M$-PAM modulation scheme, $P(\gamma)$ is given by [13]

$$
P(\gamma)=C Q(A \sqrt{\gamma})
$$

where $Q(\cdot)$ is the Gaussian $Q$-function and $A, C$ are modulation-specific constants given in [13, pp. 224-225, 252 ]. For integer $m$, it is shown that [13, pp. 127 ]

$$
\begin{aligned}
L(A, b, m)= & \int_{0}^{\infty} f(\gamma, b, m) Q(A \sqrt{\gamma}) d \gamma= \\
& \frac{1}{2}\left[1-\mu \sum_{l=0}^{m-1}\left(\begin{array}{c}
2 l \\
l
\end{array}\right)\left(\frac{1-\mu^{2}}{4}\right)^{l}\right]
\end{aligned}
$$

where

$$
\mu=\left(A^{2}(b / 2) /\left(1+A^{2}(b / 2)\right)\right)^{1 / 2} .
$$

Then, using (27) and (30), the SEP for $M$-PAM can be expressed as

$$
\mathcal{P}_{E s}=\frac{2(M-1)}{M} \sum_{k=0}^{\infty} c_{k} L(A, 2 \beta, n / 2+k) .
$$

Additionally, from (25) and (30), after some manipulations, the truncation error bound of $P_{E_{s}}$ can be expressed in closedform as

$$
\begin{aligned}
\mathcal{E}_{P A M s}= & C c_{0} \sum_{i=1}^{n / 2} \Delta_{i} L\left(A, 2 \beta /\left(1-\lambda_{2 i-1}\right), 1\right) /\left(1-\lambda_{2 i-1}\right) \\
& -C c_{0} \sum_{i=1}^{n / 2} \Delta_{i} \sum_{l=0}^{N+n / 2-1} \lambda_{2 i-1}^{l} L(A, 2 \beta, l+1)
\end{aligned}
$$

which can be very easily evaluated. 
2) $M-Q A M$ SEP: For square $M$-QAM , $P(\gamma)$ is [13]

$$
P(\gamma)=1-[1-2 F Q(A \sqrt{\gamma})]^{2}
$$

with $F$ and $A$ defined in [13, pp. 225-227,254 ]. Thus, taking into consideration the following relation ([13, pp. 130$]$ )

$$
\begin{aligned}
H(A, b, m)= & \int_{0}^{\infty} f(\gamma, b, m) Q^{2}(a \sqrt{\gamma}) d \gamma= \\
& \frac{1}{4}-\frac{1}{\pi} \rho\left\{\left(\frac{\pi}{2}-\nu\right) \sum_{l=0}^{m-1}\left(\begin{array}{c}
2 l \\
l
\end{array}\right) \frac{1}{(4(1+c))^{l}}\right. \\
& \left.-\sin (\nu) \sum_{l=1}^{m-1} \sum_{j=1}^{l} \frac{T_{j l}}{(1+c)^{l}}[\cos (\nu)]^{2(l-j)+1}\right\}
\end{aligned}
$$

with $c, \rho, T_{j, l}$ defined in [13, pp. 130 - 131] and $\nu=\tan ^{-1} \rho$ after some manipulations, the SEP for an $M$-QAM is expressed in a compact form as

$$
\begin{gathered}
\mathcal{P}_{E s}=4\left(\frac{\sqrt{M}-1}{\sqrt{M}}\right) \sum_{k=0}^{\infty} c_{k}\{L(A, 2 \beta, n / 2+k)- \\
\left.\left(\frac{\sqrt{M}-1}{\sqrt{M}}\right) H(A, 2 \beta, n / 2+k)\right\}
\end{gathered}
$$

Similar to the PAM case, the equivalent truncation error bound in (36) can be expressed in closed-form as

$$
\begin{aligned}
\mathcal{E}_{Q A M s}= & 4 c_{0} F\left\{\sum_{i=1}^{n / 2} \Delta_{i} \frac{L\left(A, 2 \beta /\left(1-\lambda_{2 i-1}\right), 1\right)}{\left(1-\lambda_{2 i-1}\right)}\right. \\
& -\sum_{i=1}^{n / 2} \Delta_{i} \sum_{l=0}^{N+n / 2-1} \lambda_{2 i-1}^{l} L(A, 2 \beta, l+1) \\
& -F \sum_{i=1}^{n / 2} \Delta_{i} \frac{H\left(A, 2 \beta /\left(1-\lambda_{2 i-1}\right), 1\right)}{\left(1-\lambda_{2 i-1}\right)} \\
& +F \sum_{i=1}^{n / 2} \Delta_{i} \sum_{l=0}^{N+n / 2-1} \lambda_{2 i-1}^{l} H(A, 2 \beta, l+1)
\end{aligned}
$$

\section{B. BEP Expressions}

In the following, BEP expressions and truncation error bounds for PAM and square QAM are derived.

1) $M$-PAM BEP: For the calculation of BEP for an $M$-PAM system with Gray coding, $P(\gamma)$ is defined as [15]

$$
P(\gamma)=\frac{1}{\log _{2} M} \sum_{j=1}^{\log _{2} M} P_{b}(j, \gamma)
$$

where

$$
\begin{aligned}
P_{b}(j, \gamma)= & \frac{2}{M} \sum_{i=0}^{\left(1-2^{-j}\right) M-1}\left\{(-1)^{\left\lfloor i 2^{j-1} / M\right\rfloor} \times\right. \\
& \left(2^{j-1}-\left\lfloor\frac{i 2^{j-1}}{M}+\frac{1}{2}\right\rfloor\right) \times \\
& \left.Q\left((2 i+1) \sqrt{\frac{6 \log _{2} M \gamma}{\left(M^{2}-1\right)}}\right)\right\}
\end{aligned}
$$

Consequently, using (30) the BEP can be written as

$$
\mathcal{P}_{E b}=\sum_{k=0}^{\infty} \frac{c_{k}}{\log _{2} M} \sum_{j=1}^{\log _{2} M} P_{b j}(2 \beta, n / 2+k)
$$

with

$$
\begin{aligned}
P_{b j}(2 \beta, m)= & \frac{2}{M} \sum_{i=0}^{\left(1-2^{-j}\right) M-1}\left\{(-1)^{\left\lfloor i 2^{j-1} / M\right\rfloor} \times\right. \\
& \left(2^{j-1}-\left\lfloor\frac{i 2^{j-1}}{M}+\frac{1}{2}\right\rfloor\right) \times \\
& L\left(D_{i}, 2 \beta, m\right)
\end{aligned}
$$

and

$$
D_{i}=(2 i+1) \sqrt{\frac{6 \log _{2} M}{M^{2}-1}} .
$$

Its truncation error bound becomes

$$
\begin{aligned}
\mathcal{E}_{P A M b}= & \sum_{i=1}^{n / 2} \frac{c_{0} \Delta_{i}}{\log _{2} M} \sum_{j=1}^{\log _{2} M} \frac{P_{b j}\left(2 \beta /\left(1-\lambda_{2 i-1}\right), 1\right)}{\left(1-\lambda_{2 i-1}\right)}- \\
& \sum_{i=1}^{n / 2} \frac{c_{0} \Delta_{i}}{\log _{2} M} \sum_{l=0}^{N+n / 2-1} \lambda_{2 i-1}^{l} \sum_{j=1}^{\log _{2} M} P_{b j}(2 \beta, l+1)
\end{aligned}
$$

2) $M-Q A M B E P$ : It is well known that the BEP of a square $M$-QAM with Gray coding, is equal to the BEP of an $\sqrt{M}$ PAM [15]. Therefore, by substituting $M$ by $\sqrt{M}$ in (40) and (41) expressions for the BEP of $M$-QAM over Hoyt fading channels can be obtained, while similarly an expression for the truncation error can be found.

\section{Performance Evaluation and Discussion}

In this section, performance evaluation results will be presented comparing the previously derived theoretical analysis with computer simulations. In our computer simulation experiments, we have considered a $2 \times 2$ MIMO system with Alamouti space-time coding [1]. In Fig. 1, truncation error bounds of the chi-squared series PDF for two Hoyt fading scenarios are depicted. In the first scenario, the channel fading coefficients are quite similar, i.e., $\left(\Omega_{1,1}, q_{1,1}\right)=(0.25,0.9)$, $\left(\Omega_{1,2}, q_{1,2}\right)=(0.25,0.8) \quad\left(\Omega_{2,1}, q_{2,1}\right)=(0.25,0.75)$, $\left(\Omega_{2,2}, q_{2,2}\right)=(0.25,0.7)$, resulting in a small spread of the quadratic form parameters $a_{i}$ in (5). In the second scenario, a large spread is obtained by selecting the Hoyt parameters as $\left(\Omega_{1,1}, q_{1,1}\right)=(0.4,0.4),\left(\Omega_{1,2}, q_{1,2}\right)=(0.3,0.5)$ $\left(\Omega_{2,1}, q_{2,1}\right)=(0.2,0.6),\left(\Omega_{2,2}, q_{2,2}\right)=(0.1,0.8)$. The value of the parameter $\beta$ has been chosen to maximize the convergence of the chi-squared series in (7) according to the relation [10],[11]

$$
\beta=\frac{2 \max _{i}\left\{a_{i}\right\} \min _{i}\left\{a_{i}\right\}}{\max _{i}\left\{a_{i}\right\}+\min _{i}\left\{a_{i}\right\}} .
$$

Moreover, for the first scenario $N=15$ and for the second $N=20$. It can be observed from Fig. 1 that in both fading scenarios the proposed bound is meaningful and much tighter 


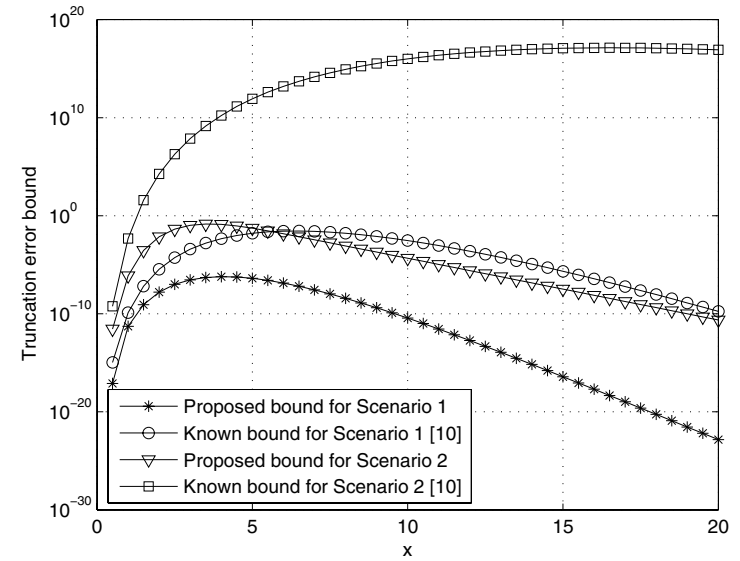

Fig. 1. Comparison of chi-squared series PDF truncation error bounds

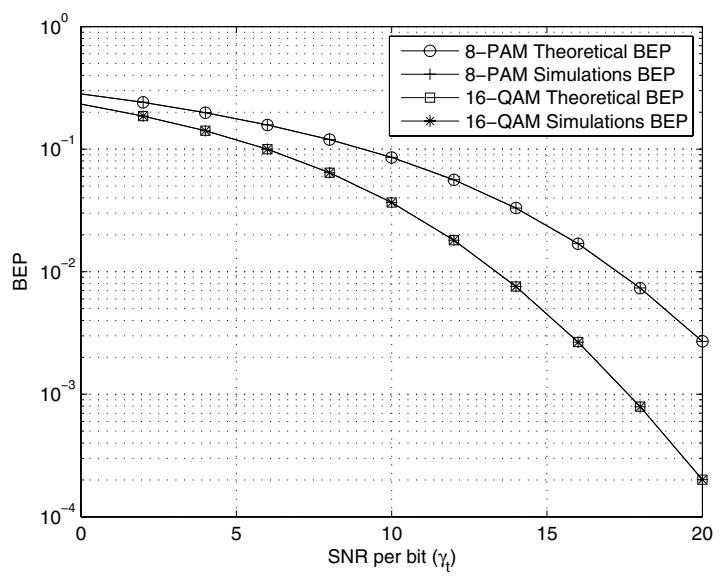

Fig. 2. BEP for OSTBC over Hoyt fading

than the bound derived in [11], with the latter appearing to be impractical for large spreads of the quadratic form parameters.

Theoretical and experimental evaluation of the BEP for 8PAM and 16-QAM for the second fading scenario is shown in Fig. 2. The theoretical curves have been obtained by retaining 21 terms from the BEP series expression in (40). It is noted that theoretical and experimental results almost coincide, thus veryfing the fast convergence of the chi-squared series expansion and the validity of proposed theoretical analysis.

In Fig. 3, the corresponding BEP truncation error bounds given by (43), for $N=15$ and $N=20$ are depicted. By comparing Figs. 2 and 3, it can be seen that for $N=20$ a very reliable bound is obtained, which is at least one order of magnitute lower than the BEP itself for all SNRs. Moreover, as shown in Fig. 3, a slight increase in the number of series terms that are retained leads to a significant improvement of the proposed bounds.

Similar performance evaluation results have been obtained using (32) and (36). However due to space limitations, these results are not presented here.

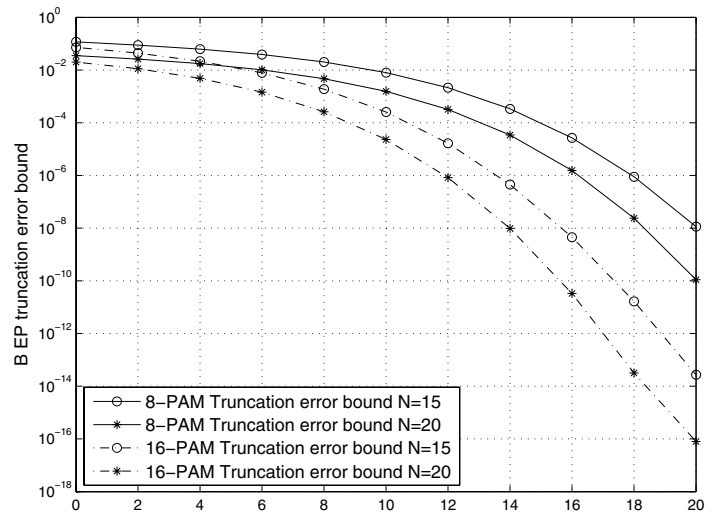

Fig. 3. Truncation error bound for the BEP for an 8-PAM and a 16-QAM

\section{REFERENCES}

[1] E. Larsson and P. Stoica, Space-Time Block Coding for Wireless Communications. New York: Cambridge University Press, 2003.

[2] M. Dohler and H. Aghvami, "Information outage probability of distributed STBCs over Nakagami fading channels," IEEE Commun. Lett., vol. 8, no. 7, pp. 437-439, Jul. 2004.

[3] G. Femenias, "BER performance of linear STBC from orthogonal designs over MIMO correlated Nakagami-m fading channels," IEEE Trans. Veh. Technol., vol. 53, no. 2, pp. 1074-1080, Mar 2004.

[4] R. U. Nabar, H. Bolcskei, and A. Paulraj, "Diversity and outage performance in space-time block coded Ricean MIMO channels," IEEE Trans. Wireless Commun., vol. 4, no. 5, pp. 2519-2532, Sept. 2005.

[5] A. Anamalai, C. Tellambura, and V. K. Bhargava, "Simple and accurate methods for the outage analysis in cellular mobile radio systems-A unified approach," IEEE Trans. Commun., vol. 49, no. 2, pp. 303-316, Feb. 2001.

[6] J. Cheng and T. Berger, "Capacity of Nakagami- $q$ (Hoyt) fading channels with channel side information," in Proc. IEEE International Conference on Communication Technology, vol. 55, Apr. 2003, pp. 1915-1918.

[7] B. Chytil, "The distribution of amplitude scintillation and the conversion of scintillation indices," J. Atmos. Terr. Phys., vol. 29, pp. 1175-1177, Sept. 1967.

[8] S. Khatalin and J. P. Fonseka, "On the channel capacity in Rician and Hoyt fading environments with MRC diversity," IEEE Trans. Veh. Technol., vol. 55, no. 1, pp. 137-141, Jan. 2006.

[9] G. A. Ropokis, A. A. Rontogiannis, and P. T. Mathiopoulos, "Information outage probability of orthogonal space-time block codes over Hoyt fading channels," in International Workshop on Satellite and Space Communications, vol. 2, Sept. 2006, pp. 135-139.

[10] H. Ruben, "Probability content of regions under spherical normal distributions, iv: The distribution of homogeneous and non-homogeneous quadratic functions of normal variables," The Annals of Mathematical Statistics, vol. 33, no. 2, pp. 542-570, Jun. 1962.

[11] S. Kotz, N. L. Johnson, and D. W. Boyd, "Series representations of distributions of quadratic forms in normal variables. i. central case," The Annals of Mathematical Statistics, vol. 38, no. 3, pp. 823-837, Jun. 1967.

[12] A. M. Mathai and S. B. Provost, Quadratic Forms in Random Variables: Theory and Applications. Marcel Dekker, Inc, 1992.

[13] M. K. Simon and M.-S. Alouini, Digital Communication over Fading Channels. John Wiley and Sons, 2005.

[14] C.-X. Wang, N. Youssef, and M. Paetzold, "Level-crossing rate and average duration of fades of deterministic simulation models for NakagamiHoyt fading channels," in the 5th International Symposium on Wireless Personal Multimedia Communications, vol. 1, Oct. 2002, pp. 272-276.

[15] K. Cho and D. Yoon, "On the general BER expression of one- and twodimensional amplitude modulations," IEEE Trans. Commun., vol. 50, no. 7, pp. 1074-1080, Jul 2002. 